\section{PTH-130 THE EFFICACY OF TWO COMMERCIALLY AVAILABLE SERUM CALPROTECTIN ASSAYS TO ASSESS DISEASE ACTIVITY IN IBD}

\begin{abstract}
${ }^{1}$ Sarah Townsend*, ${ }^{2}$ Michael Udegbune, ${ }^{1}$ Rachel Molyneux, ${ }^{1}$ Helen Steed, ${ }^{1}$ Ashit Shah, 2,3 Rousseau Gama, ${ }^{2}$ Ben Allen1, Hayley Sharrod-cole, 'Mohmad Shakil Kathawala, ${ }^{1}$ Venkata Maddirala, ${ }^{2}$ Clare Ford, ${ }^{1}$ Yaqsha Hussain, ${ }^{1,3}$ Matthew Brookes. ${ }^{1}$ New Cross Hospital, Wolverhampton, Wolverhampton, UK; ${ }^{2}$ Clinical Chemistry Department, Royal Wolverhampton NHS Trust, Wolverhampton, UK; ${ }^{3}$ University of Wolverhampton, Wolverhampton, UK
\end{abstract}

\subsection{6/gutjnl-2019-BSGAbstracts. 189}

Background Biomarkers of disease activity in IBD have variable performance when it comes to sensitivity and specificity. The clinical use of serum calprotectin remains unclear, but commercially available assays are now available. Previous studies have suggested that serum calprotectin (s-Cp) may have a role in managing IBD. Studies have shown an association between s-CP and the recurrence of Crohn's disease in the STORI cohort. ${ }^{1}$ These studies did however show that $s-C p$ has a similar profile in $\mathrm{CD}$ to that offered by C-reactive protein. Others have investigated its role in the unselected evaluation of Gastroenterology patients and found that its performance in these cases is disappointing and unlikely to be of value. ${ }^{2}$ There remains uncertainty about the role of $s-\mathrm{Cp}$ in the management of patients with IBD.

Design Patients attending the IBD clinic between July 2017 and December 2017 were assessed and standard blood tests and a faecal calprotectin were collected. Serum was analysed using two commercially available calprotectin assays (Buhlmann and Immunodiagnostics). Additional data recorded included demographics, disease classification and an assessment of the clinical interpretation of their disease activity.

Results There was no difference seen in s-Cp in UC patients comparing active vs quiescent UC, except in those with pancolitis (Montreal E3) using the Buhlmann assay (median 5098 vs $3502 \mathrm{ng} / \mathrm{mL} ; \mathrm{p}=0.0362)$.

Median s-Cp was, however, significantly higher in patients with active $C D$ than in those with quiescent $C D$ for both assays $(5507$ vs $3830 \mathrm{ng} / \mathrm{mL} ; \mathrm{p}=0.0001$ (Buhlmann assay) and 5131 vs $2994 \mathrm{ng} / \mathrm{mL}$; p $=0.0003$ (Immunodiagnostics)). The difference in s-Cp remained significant in patients with both small $(\mathrm{p}=0.008)$ and large bowel CD $(\mathrm{p}=0.005)$, with similar results for each serological test.

Serum calprotectin correlated with faecal calprotectin in all patients with IBD $(\mathrm{r}=0.2362 ; \mathrm{p}=0.0051$ (Buhlmann) and $\mathrm{r}=0.2183 ; \mathrm{p}=0.0098$ (Immunodiagnostics)).

Similarly, s-Cp correlated with serum CRP in individuals with IBD and specifically CD $(r=0.4865$; $p<0.0001$ (Buhlmann) and $r=0.5016 ; p<0.0001$ (Immunodiagnostics)).

Conclusions There does not appear to be any significant association with the confirmation of ulcerative colitis disease activity. In patients with $\mathrm{CD}$ there appears to be a significant association between s-Cp, for both commercially available assays, and the presence of active disease irrespective of the disease location.

S-Cp also appears to significantly correlate positively with other markers of disease activity in patients with active and inactive IBD. Additional data analysis suggests that $\mathrm{s}$-Cp offers a potential benefit over currently available biomarkers, specifically in patients with CD.

\section{REFERENCE}

1. http://dx.doi.org/10.1016/j.crohns.2013.06.008

2. https://doi.org/10.1016/j.clinbiochem.2017.01.006

\section{PTH-131 MULTI-CENTRE COMPARATIVE EFFECTIVENESS OF VEDOLIZUMAB AND USTEKINUMAB AS INDUCTION THERAPY IN ANTI-TNF REFRACTORY CROHN'S DISEASE}

Royal Liverpool University Hospital Tristan Townsend ${ }^{1 *}$, ${ }^{2}$ Violeta Razanskaite, ${ }^{1}$ Stephanie Michail, ${ }^{1}$ James Morgan, ${ }^{1}$ Michael Davies, ${ }^{1}$ Daneil Storey, ${ }^{3}$ Christopher Watters, ${ }^{3}$ Douglas Penman, ${ }^{4}$ Mira Swaminathan, ${ }^{3}$ Joseph Sabine, ${ }^{2}$ Adam Chapman, ${ }^{4}$ Ian Reilly, ${ }^{3}$ Paul Flanagan, ${ }^{2}$ Keith Bodger, ${ }^{1}$ Sreedhar Subramanian. ${ }^{1}$ Royal Liverpool University Hospital, Liverpool, UK; ${ }^{2}$ Aintree University Hospital, Liverpool, UK; ${ }^{3}$ Arrowe Park Hospital, Upton, UK; ${ }^{4}$ Countess of Chester Hospital, Chester, UK

\subsection{6/gutjnl-2019-BSGAbstracts.190}

Introduction Anti-tumour necrosis factor (TNF) agents are effective in Crohn's disease (CD), but up to 30\% of patients fail to respond or develop intolerance and require alternative biological therapy. Both vedolizumab and ustekinumab are licensed to treat anti-TNF refractory CD patients. Clinical trials of vedolizumab and ustekinumab in anti-TNF refractory patients seem to suggest comparable efficacy, but no real life data exist to enable clinicians to guide decision-making. We conducted a multi-centre retrospective cohort study to assess the comparative effectiveness of vedolizumab and ustekinumab in treating anti-TNF refractory CD.

Methods CD patients who were commenced on vedolizumab or ustekinumab following exposure to anti-TNF therapy were included. Disease activity was monitored serially by calculation of Harvey-Bradshaw index (HBI) for up to 4 months. Faecal calprotectin (FC) at baseline and subsequent visits were recorded if available. Clinical response was defined as decrease in $\mathrm{HBI} \geq 3$ and remission by $\mathrm{HBI}<5$. We compared the effectiveness of ustekinumab and vedolizumab.

Results After exclusion of patients without evaluable data, 51 patients commencing vedolizumab and 25 commencing ustekinumab therapy were included. Baseline characteristics (age, disease location, behaviour, smoking status and baseline FC) were comparable in both cohorts. Clinical response, remission and steroid-free remission rates were comparable between vedolizumab and ustekinumab at 2 and 4 months (see table 1). There was a significant reduction in HBI for vedolizumab at 2 months $(1.8,95 \%$ CI 0.50 to $3.13, \mathrm{p}=0.008)$ and ustekinumab at 4 months $(3.4,95 \%$ CI 1.84 to $4.96, \mathrm{p}=$ 0.0001).

\begin{tabular}{lllll} 
Abstract PTH-131 Table 1 & & & \\
\hline & & Vedolizumab & Ustekinumab & $\begin{array}{l}\text { Fisher's Exact } \\
\text { Test (P value) }\end{array}$ \\
\hline Response & 2 months & $37 \%$ & $44 \%$ & 0.623 \\
& 4 months & $49 \%$ & $60 \%$ & 0.465 \\
Remission & 2 months & $18 \%$ & $28 \%$ & 0.372 \\
& 4 months & $27 \%$ & $40 \%$ & 0.302 \\
Steroid-Free & 2 months & $12 \%$ & $20 \%$ & 0.489 \\
Remission & 4 months & $25 \%$ & $36 \%$ & 0.422 \\
\hline
\end{tabular}

Conclusions In a multi-centre cohort of anti-TNF refractory $\mathrm{CD}$ patients, vedolizumab and ustekinumab appear equally effective in the short-term. Our observations warrant longerterm follow up and further validation in independent cohorts. 